\title{
Generalized Goldstone Theorem: Automatic Imposition of the Higgs Mechanism and Application to Scale and Conformal Symmetry Breaking
}

\author{
A. Chodos* \\ Physics Department, Yale University, New Haven, CT 06520-8120 \\ G. Gallatin ${ }^{\dagger}$ \\ Bell Labs, Lucent Technologies, \\ Murray Hill, NJ 07974-0636
}

November 19, 2018

\begin{abstract}
Standard discussions of Goldstone's theorem based on a symmetry of the action assume constant fields and global transformations, i.e., transformations which are independent of spacetime coordinates. By allowing for arbitrary field distributions in a general representation of the symmetry we derive a generalization of the standard Goldstone's theorem. When applied to gauge bosons coupled to scalars with a spontaneously broken symmetry the generalized theorem automatically imposes the Higgs mechanism, i.e., if the expectation value of the scalar field is nonzero then the gauge bosons must be massive. The other aspect of the Higgs mechanism, the disappearance of the "would be" Goldstone boson, follows directly from the generalized symmetry condition itself. We also use our generalized Goldstone's theorem to analyze the case of a system in which scale and conformal symmetries are both spontaneously broken.
\end{abstract}

\section{Introduction}

Symmetry, symmetry breaking, Goldstone bosons and the Higgs mechanism play a very important role in modern physics. (See for example [1] [2] [3] 仼) Here we present a more general approach to these ideas which explores the consequences of Goldstone's theorem for space-time and gauge symmetries and

\footnotetext{
*Present Address, American Physical Society, Washington, DC, chodos@aps.org

${ }^{\dagger}$ gallatin@lucent.com
} 
shows that the Higgs mechanism is not as "mysterious" or "miraculous" as it is sometimes presented to be. We also resolve some old questions regarding the the breaking of scale and conformal symmetry.

We consider the physical consequences of actions which have a symmetry or a set of symmetries. Precisely what we mean by this is discussed in detail below. For concreteness we work out the details for the classical action of a set of fields $\Phi_{a}$, which we denote by $S[\Phi]$. To treat the quantum theory one computes the functional integral over all field configurations (in a given function space) of $\exp (i S[\Phi]+i J \cdot \Phi)$ to obtain the generating functional $Z[J]$. Here the dot indicates the appropriate inner product over spacetime position, spacetime indices and internal indices. The effective action $\Gamma[\Phi]$, 迎[2] 3] a Legendre transformation of $\ln (Z[J])$, is the analog of $S[\Phi]$ but includes all quantum effects. That is, whereas $\delta S / \delta \Phi(x)=0$ is the equation of motion for the classical field configuration $\Phi_{C}(x), \delta \Gamma / \delta \Phi(x)=0$ is the equation of motion for the vacuum expectation value of the field $\langle\Phi(x)\rangle$. Indeed the $n^{t h}$ functional derivative of $\Gamma[\Phi]$ is the $n$-point quantum Greens function. Generally, but certainly not always, $\Gamma[\Phi]$ will have the same symmetry properties as $S[\Phi]$. To lowest or "tree" order the two actions coincide and in a sense $\Gamma[\Phi]$ can be thought of as just a more complicated functional of $\Phi$ than $S[\Phi]$. Hence our approach applies to both $S[\Phi]$ and to $\Gamma[\Phi]$ for any given symmetry that holds for either functional. We will generally assume that $\Phi$ is classically a commuting field or quantum mechanically a bosonic field, but the same approach can be applied to classical Grassmann fields or to quantum Fermionic fields yielding similar results with, of course, the requisite care in factor ordering.

\section{General Symmetry Condition}

Consider a Lagrangian density $\mathcal{L}(\Phi(x), \partial \Phi(x))$ which depends on a set of fields $\Phi_{a}$ and their first derivatives, $\partial_{\mu} \Phi_{a}$. Here $a$ can be an internal index, a spacetime index or a combination of the two. The action is defined by

$$
S[\Phi]=\int d^{D} x \mathcal{L}(\Phi(x), \partial \Phi(x))
$$

and is taken to be invariant, i.e., $S[\Phi]=S\left[\Phi^{\prime}\right]$, under a continuous set of transformations of the fields given by

$$
\begin{aligned}
\Phi_{a}(x) \rightarrow \Phi_{a}^{\prime}(x) & =F(\Phi(x), \partial \Phi(x), \ldots, x) \\
& =\Phi_{a}(x)+\Delta_{a}(\Phi(x), \partial \Phi(x), \ldots, x)+\cdots
\end{aligned}
$$

where the last expression is the infinitesimal form of the transformation, i.e, $\Delta_{a} \ll 1$. In terms of the Lagrangian this symmetry has the form

$$
\begin{aligned}
\int d^{D} x \mathcal{L} & (\Phi(x), \partial \Phi(x)) \\
= & \int d^{D} x \mathcal{L}\left(\Phi^{\prime}(x), \partial \Phi^{\prime}(x)\right)
\end{aligned}
$$




$$
=\int d^{D} x \mathcal{L}(F(\Phi(x), \partial \Phi(x), \ldots, x), \partial F(\Phi(x), \partial \Phi(x), \ldots, x))
$$

We have assumed the change in $\Phi_{a}$ may depend locally on $\Phi_{a}$ and possibly explicitly on $x$ as well. For ease of notation we will abbreviate the $\Phi$ dependence of $\mathcal{L}, F$ and $\Delta$ as

$$
\begin{aligned}
\mathcal{L}(\Phi(x), \partial \Phi(x)) & \equiv \mathcal{L}[\Phi(x)] \\
F_{a}(\Phi(x), \partial \Phi(x), \ldots, x) & \equiv F_{a}[\Phi(x), x] \\
\Delta_{a}(\Phi(x), \partial \Phi(x), \ldots, x) & \equiv \Delta_{a}[\Phi(x), x]
\end{aligned}
$$

Infinitesimally a symmetry is simply the statement that the gradient of the action at any point in function space is perpendicular to the direction defined by $\Delta_{a}$, i.e,

$$
\int d^{D} x \frac{\delta S[\Phi]}{\delta \Phi_{a}(x)} \Delta_{a}[\Phi(x), x]=0
$$

The symmetry condition expressed in (5) is not an equation for $\Phi$ and in fact must hold for all values of $\Phi$. Thus all the functional derivatives of (5) with repect to $\Phi$ must vanish. This condition effectively assumes the equivalent of analyticity of $S[\Phi]$ in function space. If the same idea is applied to $\Gamma[\Phi]$, the effective action computed by evaluating a functional integral, then the vanishing of the various functional derivatives are termed "generalized WardTakahashi identities". The various ways in which (5) can be satisfied, which lead to Noether's theorem and the distinction between internal and external symmetries are discussed in the Appendix.

Taking one functional derivative of the symmetry condition (5) and evaluating it at the equation of motion yields, as discussed below, what can be seen as a generalized Goldstone theorem

$$
\int d^{D} x^{\prime}\left(\frac{\delta^{2} S}{\delta \Phi_{a}(x) \delta \Phi_{b}\left(x^{\prime}\right)} \Delta_{a}\left[\Phi\left(x^{\prime}\right), x^{\prime}\right]\right)_{\Phi_{C}}=0
$$

Substituting (1) and assuming locality of $\Delta_{a}$ as expressed in (4) yields the following after some simple manipulations

$$
\begin{gathered}
{\left[-\partial_{\alpha}\left(\frac{\partial^{2} \mathcal{L}}{\partial\left(\partial_{\alpha} \Phi_{a}\right) \partial\left(\partial_{\mu} \Phi_{b}\right)} \partial_{\mu} \Delta_{b}\right)-\partial_{\alpha}\left(\frac{\partial^{2} \mathcal{L}}{\partial\left(\partial_{\alpha} \Phi_{a}\right) \partial \Phi_{b}} \Delta_{b}\right)\right.} \\
\left.+\frac{\partial^{2} \mathcal{L}}{\partial \Phi_{a} \partial\left(\partial_{\mu} \Phi_{b}\right)} \partial_{\mu} \Delta_{b}+\frac{\partial^{2} \mathcal{L}}{\partial \Phi_{a} \partial \Phi_{b}} \Delta_{b}\right]_{\Phi_{C}}=0
\end{gathered}
$$

The remainder of the paper explores some of the consequences of this equation.

\section{Goldstone's Theorem}

There are nominally two consequences of Goldstone's theorem. The primary one is the requirement for the existence of some number of massless bosons, called Goldstone bosons, in the theory if the symmetry is spontaneously broken. 
The secondary condition is that the Goldstone bosons decouple from the other degrees of freedom in the limit of zero momentum.

Symmetry breaking is based on the additive nature of (2) which indicates that a given field configuration is not invariant under the symmetry transformation. In particular it is often the case that $\Delta_{a} \equiv 0$ if and only if $\Phi_{a}=0$ and so for any nonzero field configuration the transformation in (2) is inhomogeneous and the symmetry is spontaneously broken, i.e., a nonzero field configuration is not invariant under the symmetry transformation whereas the zero field configuration is invariant.

We begin by reviewing the standard approach to Goldstones theorem as given for example in the book by Peskin and Schroeder [1] This form of the derivation proceeds by considering constant fields and field transformations for Lagrangians of the form

$$
\mathcal{L}=T(\partial \Phi)-V(\Phi)
$$

Specializing to constant fields the equations of motion reduce to

$$
\left.\frac{\partial V}{\partial \Phi_{a}}\right|_{\Phi_{C}}=0
$$

which shows that the constant field $\Phi_{C}$ is an extremum, commonly a minimum, of $V$. Expanding $V$ about this miminum yields

$$
V(\Phi)=V\left(\Phi_{C}\right)+\frac{1}{2}\left(\Phi-\Phi_{C}\right)_{a}\left(\Phi-\Phi_{C}\right)_{b}\left(\frac{\partial^{2} V}{\partial \Phi_{a} \partial \Phi_{b}}\right)_{\Phi_{C}}+\ldots
$$

The coefficient of the quadratic term is a symmetric matrix, known as the mass matrix, whose eigenvalues are the square of the masses of the various fields obtained from $\left(\Phi-\Phi_{C}\right)_{a}$ after applying the linear transformation which diagonalizes the matrix. Since the symmetry condition is taken to hold for arbitrary field configurations it holds as well for constant fields in which case the potential $V$ itself is invariant, i.e.,

$$
V(\Phi)=V(\Phi+\Delta)
$$

which implies

$$
\frac{\partial V}{\partial \Phi_{a}} \Delta_{a}(\Phi)=0
$$

Now differentiate with respect to $\Phi_{b}$ and evaluate the result at $\Phi_{C}$ to obtain

$$
\left(\frac{\partial^{2} V}{\partial \Phi_{b} \partial \Phi_{a}}\right)_{\Phi_{C}} \Delta_{a}\left(\Phi_{C}\right)=0
$$

This shows that the mass matrix has a zero eigenvalue for each linearly independent symmetry vector $\Delta\left(\Phi_{C}\right)$ satisfying the above equation. The number of linearly independent nonzero vectors $\Delta\left(\Phi_{C}\right)$ is referred to as the number of broken generators, $N_{B}$, and hence there is one Goldstone boson for each broken generator. Our more general result (6) or equivalently (7) yields this same 
condition as well if we assume Lagrangians of the form given in (8) and set $\Phi_{C}$ to a constant field in equation (6) or (7) which can now clearly be seen as a generalization of the above equation.

The decoupling follows from considering a change of variables which diagonalizes the mass matrix. This can be done by writing

$$
\Phi_{a}=\Phi_{a}\left(\xi_{i}, \rho_{j}\right)
$$

where $i=1, \ldots, N_{B}$ and $j=1, \ldots, N-N_{B}$ with $\xi_{i}$ and $\rho_{j}$ defined implicitly by choosing $\Phi_{a}\left(\xi_{i}, \rho_{j}\right)$ so that

$$
\begin{aligned}
\left(\begin{array}{ll}
\frac{\partial^{2} V(\Phi(\xi, \rho))}{\partial \xi_{i} \partial \xi_{i^{\prime}}} & \frac{\partial^{2} V(\Phi(\xi, \rho))}{\partial \xi_{i} \partial \rho_{j^{\prime}}} \\
\frac{\partial^{2} V(\Phi(\xi, \rho))}{\partial \rho_{j} \partial \xi_{i^{\prime}}} & \frac{\partial^{2} V(\Phi(\xi, \rho))}{\partial \rho_{j} \partial \rho_{j^{\prime}}}
\end{array}\right)_{\Phi_{C}} & =\left(\begin{array}{cc}
\frac{\partial \Phi_{a}}{\partial \xi_{i}} \frac{\partial^{2} V}{\partial \Phi_{a} \partial \Phi_{b}} \frac{\partial \Phi_{b}}{\partial \xi_{\xi^{\prime}}} & \frac{\partial \Phi_{a}}{\partial \xi_{i}} \frac{\partial^{2} V}{\partial \Phi_{a} \partial \Phi_{b}} \frac{\partial \Phi_{b}}{\partial \rho_{j^{\prime}}} \\
\frac{\partial \Phi_{a}}{\partial \rho_{j}} \frac{\partial^{2} V}{\partial \Phi_{a} \partial \Phi_{b}} \frac{\partial \Phi_{b}}{\partial \xi_{i^{\prime}}} & \frac{\partial \Phi_{a}}{\partial \rho_{j}} \frac{\partial^{2} V}{\partial \Phi_{a} \partial \Phi_{b}} \frac{\partial \Phi_{b}}{\partial \rho_{j^{\prime}}}
\end{array}\right)_{\Phi_{C}}\left(\begin{array}{cc}
0 \\
0 & 0 \\
0 & -\left(m_{j}\left(\Phi_{C}\right)\right)^{2} \delta_{j j^{\prime}}
\end{array}\right) \\
& =\left(\begin{array}{cc}
0 \\
0
\end{array}\right)
\end{aligned}
$$

where there is no sum on $j$ in the last matrix. The $\xi_{i}$ are the Goldstone bosons (the $\xi \xi$ sector of the mass matrix vanishes by definition) and the $\rho_{i}$ are the remaining bosons, i.e., at any given value of $\Phi_{C}$ the $\xi_{i}$ are tangent to the symmetry directions at that point in function space whereas the $\rho_{j}$ are perpendicular to these directions. The symmetry transformation (5) becomes $\xi_{i} \rightarrow \xi_{i}+\delta \xi_{i}$, $\rho_{j} \rightarrow \rho_{j}$ for $\Phi_{a} \rightarrow \Phi_{a}+\Delta_{a}(\Phi)$ or equivalently

$$
\Delta_{a}(\Phi)=\frac{\partial \Phi_{a}}{\partial \xi_{i}} \delta \xi_{i}
$$

Note that $\delta \xi_{i}$ must be constant for $\Delta(\Phi)$ constant. The symmetry condition (5) in terms of the new variables yields

$$
\begin{aligned}
0 & =\frac{\delta \mathcal{L}[\Phi(\xi, \rho)]}{\delta \xi_{i}} \delta \xi_{i} \\
& =\frac{\partial \mathcal{L}[\Phi(\xi, \rho)]}{\partial \xi_{i}} \delta \xi_{i} \\
& =\frac{\partial \mathcal{L}^{\prime}(\xi, \rho)}{\partial \xi_{i}} \delta \xi_{i}
\end{aligned}
$$

where the $\partial_{\mu} \delta \xi_{i}$ term vanishes since $\delta \xi_{i}$ must be constant for the symmetry to hold. Hence $\partial \mathcal{L}^{\prime} / \partial \xi_{i}=0$ and $\mathcal{L}^{\prime}$ can depend only on $\partial_{\mu} \xi_{i}$. Since $\partial_{\mu} \xi_{i}$ vanishes in the limit of zero momentum the Goldstone bosons vanish or decouple in this limit

\section{Automatic Higgs}

Consider a $U(1)$ gauge model in which a complex scalar field $\phi=\phi_{1}+i \phi_{2}$ with $\phi_{i}$ real, $i=1,2$, is coupled in a locally gauge invariant way to a $U(1)$ gauge 
field, $A_{\mu}$. In this case

$$
\begin{aligned}
\Phi_{a} & \rightarrow\left(A_{\mu}, \phi_{i}\right) \\
\Delta_{a} & \rightarrow\left(\partial_{\mu} \theta, \varepsilon_{i j} \phi_{j} \theta\right)
\end{aligned}
$$

with $\theta$ an arbitrary infinitesimal scalar function of position. Note we are working in units with the electric charge $e=1$. Take the Lagrangian to have the form

$$
\begin{aligned}
\mathcal{L} & =\mathcal{L}_{A}\left(\partial_{\mu} A_{\nu}\right)+\mathcal{L}_{\phi}\left(\partial_{\mu} \phi_{i}, \phi_{i}\right)+\mathcal{L}_{A \phi}\left(A_{\mu}, \partial_{\mu} \phi_{i}, \phi_{i}\right) \\
& =-\frac{1}{4} F_{\mu \nu} F^{\mu \nu}+\partial_{\mu} \phi_{i} \partial^{\mu} \phi_{i}-V\left(\phi_{i} \phi_{i}\right)+\mathcal{L}_{A \phi}\left(A_{\mu}, \partial_{\mu} \phi_{i}, \phi_{i}\right)
\end{aligned}
$$

where $F_{\mu \nu}=\partial_{\mu} A_{\nu}-\partial_{\nu} A_{\mu}$ so that $\mathcal{L}_{A}$ is invariant under gauge transformations and $\mathcal{L}_{\phi}$ is invariant under $\phi \rightarrow e^{i \theta} \phi$ for $\theta$ constant. The extra term $\mathcal{L}_{A \phi}$ is explicitly included to "boost" the symmetry from a global one to a local one. It allows for terms of the form $A \phi \partial \phi$ and $A A \phi \phi$ which are the lowest order terms with saturated indices. That is for arbitrary, i.e., not necessarilly infinitesimal $\theta$ the full Lagrangian is required to be symmetric under

$$
\begin{aligned}
A_{\mu} & \rightarrow A_{\mu}+\partial_{\mu} \theta \\
\phi & \rightarrow e^{i \theta} \phi
\end{aligned}
$$

We explicitly assume the only $\partial \phi \partial \phi$ term in $\mathcal{L}$ is the standard kinetic energy term from $\mathcal{L}_{\phi}$.

For arbitrary infinitesimal functions $\theta$ local gauge invariance yields the following symmetry condition for the action

$$
0=\int d^{D} x \frac{\delta S}{\delta A_{\beta}} \partial_{\beta} \theta+\int d^{D} x \frac{\delta S}{\delta \phi_{j}} \varepsilon_{j k} \phi_{k} \theta
$$

Note that $\mathcal{L}$ is locally gauge invariant and not just $S$ thus the remaining $\int d^{D} x$ integration in the above equation can be dropped as discussed in previous sections. The generalized symmetry equation yields two distinct equations since we can take derivatives with respect to $A$ and $\phi$

$$
\begin{aligned}
0= & \left(\int d^{D} x \frac{\delta^{2} S}{\delta A_{\alpha} \delta A_{\beta}} \partial_{\beta} \theta+\int d^{D} x \frac{\delta^{2} S}{\delta A_{\alpha} \delta \phi_{j}} \varepsilon_{j k} \phi_{k} \theta\right)_{\Phi_{C}} \\
= & -\partial_{\nu}\left(\frac{\partial^{2} \mathcal{L}}{\partial\left(\partial_{\nu} A_{\alpha}\right) \partial\left(\partial_{\mu} A_{\beta}\right)} \partial_{\mu} \partial_{\beta} \theta\right)+\frac{\partial^{2} \mathcal{L}}{\partial A_{\alpha} \partial\left(\partial_{\mu} A_{\beta}\right)} \partial_{\mu} \partial_{\beta} \theta \\
& -\partial_{\nu}\left(\frac{\partial^{2} \mathcal{L}}{\partial\left(\partial_{\nu} A_{\alpha}\right) \partial A_{\beta}} \partial_{\beta} \theta\right)+\frac{\partial^{2} \mathcal{L}}{\partial A_{\alpha} \partial A_{\beta}} \partial_{\beta} \theta \\
& -\partial_{\nu}\left(\frac{\partial^{2} \mathcal{L}}{\partial\left(\partial_{\nu} A_{\alpha}\right) \partial\left(\partial_{\mu} \phi_{j}\right)} \partial_{\mu} \varepsilon_{j k} \phi_{k} \theta\right)+\frac{\partial^{2} \mathcal{L}}{\partial A_{\alpha} \partial\left(\partial_{\mu} \phi_{j}\right)} \partial_{\mu} \varepsilon_{j k} \phi_{k} \theta \\
& -\partial_{\nu}\left(\frac{\partial^{2} \mathcal{L}}{\partial\left(\partial_{\nu} A_{\alpha}\right) \partial \phi_{j}} \varepsilon_{j k} \phi_{k} \theta\right)+\frac{\partial^{2} \mathcal{L}}{\partial A_{\alpha} \partial \phi_{j}} \varepsilon_{j k} \phi_{k} \theta
\end{aligned}
$$


and

$$
\begin{aligned}
0= & \left(\int d^{D} x \frac{\delta^{2} S}{\delta \phi_{i} \delta A_{\beta}} \partial_{\beta} \theta+\int d^{D} x \frac{\delta^{2} S}{\delta \phi_{i} \delta \phi_{j}} \varepsilon_{j k} \phi_{k} \theta\right)_{\Phi_{C}} \\
= & -\partial_{\nu}\left(\frac{\partial^{2} \mathcal{L}}{\partial\left(\partial_{\nu} \phi_{i}\right) \partial\left(\partial_{\mu} A_{\beta}\right)} \partial_{\mu} \partial_{\beta} \theta\right)+\frac{\partial^{2} \mathcal{L}}{\partial \phi_{i} \partial\left(\partial_{\mu} A_{\beta}\right)} \partial_{\mu} \partial_{\beta} \theta \\
& -\partial_{\nu}\left(\frac{\partial^{2} \mathcal{L}}{\partial\left(\partial_{\nu} \phi_{i}\right) \partial A_{\beta}} \partial_{\beta} \theta\right)+\frac{\partial^{2} \mathcal{L}}{\partial \phi_{i} \partial A_{\beta}} \partial_{\beta} \theta \\
& -\partial_{\nu}\left(\frac{\partial^{2} \mathcal{L}}{\partial\left(\partial_{\nu} \phi_{i}\right) \partial\left(\partial_{\mu} \phi_{j}\right)} \varepsilon_{j k} \partial_{\mu}\left(\phi_{k} \theta\right)\right)+\frac{\partial^{2} \mathcal{L}}{\partial \phi_{i} \partial\left(\partial_{\beta} \phi_{j}\right)} \varepsilon_{j k} \partial_{\beta}\left(\phi_{k} \theta\right) \\
& -\partial_{\nu}\left(\frac{\partial^{2} \mathcal{L}}{\partial\left(\partial_{\nu} \phi_{i}\right) \partial \phi_{j}} \varepsilon_{j k} \phi_{k} \theta\right)+\frac{\partial^{2} \mathcal{L}}{\partial \phi_{i} \partial \phi_{j}} \varepsilon_{j k} \phi_{k} \theta
\end{aligned}
$$

To simplify notation in both equation we have implicitly assumed that the results have been evaluated at at solution to the equations of motion.

In (22) the first term vanishes identically, the second and third terms vanish because we have not allowed for any derivative coupling of the gauge fields. The fifth term and seventh terms vanish since there are no $\phi \partial A$ or $\partial A \partial \phi$ terms. In (23) the first and second terms vanish. Using the fact that $\partial_{\mu} \phi_{i} \partial^{\mu} \phi_{i}=$ $g^{\mu \nu} \delta_{i j} \partial_{\mu} \phi_{i} \partial_{\nu} \phi_{j}$ is the only term quadratic in the derivatives of $\phi$ and expanding out all the derivatives of product terms we obtain

$$
\begin{aligned}
0= & \left(\frac{\partial^{2} \mathcal{L}}{\partial A_{\alpha} \partial A_{\beta}}+\frac{\partial^{2} \mathcal{L}}{\partial A_{\alpha} \partial\left(\partial_{\beta} \phi_{j}\right)} \varepsilon_{j k} \phi_{k}\right) \partial_{\beta} \theta \\
& +\left(\frac{\partial^{2} \mathcal{L}}{\partial A_{\alpha} \partial \phi_{j}} \varepsilon_{j k} \phi_{k}+\frac{\partial^{2} \mathcal{L}}{\partial A_{\alpha} \partial\left(\partial_{\beta} \phi_{j}\right)} \varepsilon_{j k} \partial_{\beta} \phi_{k}\right) \theta
\end{aligned}
$$

and

$$
\begin{aligned}
0= & -\left(\frac{\partial^{2} \mathcal{L}}{\partial\left(\partial_{\nu} \phi_{i}\right) \partial A_{\beta}}+2 g^{\nu \beta} \varepsilon_{i k} \phi_{k}\right) \partial_{\nu} \partial_{\beta} \theta \\
+ & \left(\begin{array}{c}
\frac{\partial^{2} \mathcal{L}}{\partial \phi_{i} \partial A_{\beta}}-\partial_{\nu}\left(\frac{\partial^{2} \mathcal{L}}{\partial\left(\partial_{\nu} \phi_{i}\right) \partial A_{\beta}}\right)-4 \varepsilon_{i k} \partial^{\beta} \phi_{k} \\
+\left(\frac{\partial^{2} \mathcal{L}}{\partial \phi_{i} \partial\left(\partial_{\beta} \phi_{j}\right)}-\frac{\partial^{2} \mathcal{L}}{\partial\left(\partial_{\beta} \phi_{i}\right) \partial \phi_{j}}\right) \varepsilon_{j k} \phi_{k}
\end{array}\right) \partial_{\beta} \theta \\
& +\left(\begin{array}{c}
-2 \varepsilon_{i k} \partial^{2} \phi_{k}+\left(\frac{\partial^{2} \mathcal{L}}{\partial \phi_{i} \partial\left(\partial_{\beta} \phi_{j}\right)}-\frac{\partial^{2} \mathcal{L}}{\partial\left(\partial_{\beta} \phi_{i}\right) \partial \phi_{j}}\right) \varepsilon_{j k} \partial_{\beta} \phi_{k} \\
+\left(\frac{\partial^{2} \mathcal{L}}{\partial \phi_{i} \partial \phi_{j}}-\partial_{\nu}\left(\frac{\partial^{2} \mathcal{L}}{\partial\left(\partial_{\nu} \phi_{i}\right) \partial \phi_{j}}\right)\right) \varepsilon_{j k} \phi_{k}
\end{array}\right) \theta
\end{aligned}
$$

Since $\theta$ is an arbitrary function coefficients of $\theta, \partial \theta$, and $\partial \partial \theta$ must vanish independently which yields the following set of equations

$$
0=\frac{\partial^{2} \mathcal{L}}{\partial A_{\alpha} \partial A_{\beta}}+\frac{\partial^{2} \mathcal{L}}{\partial A_{\alpha} \partial\left(\partial_{\beta} \phi_{j}\right)} \varepsilon_{j k} \phi_{k}
$$




$$
\begin{aligned}
& 0=\frac{\partial^{2} \mathcal{L}}{\partial A_{\alpha} \partial \phi_{j}} \varepsilon_{j k} \phi_{k}+\frac{\partial^{2} \mathcal{L}}{\partial A_{\alpha} \partial\left(\partial_{\beta} \phi_{j}\right)} \varepsilon_{j k} \partial_{\beta} \phi_{k} \\
& 0=\frac{\partial^{2} \mathcal{L}}{\partial\left(\partial_{\nu} \phi_{i}\right) \partial A_{\beta}}+2 g^{\nu \beta} \varepsilon_{i k} \phi_{k} \\
& 0=\frac{\partial^{2} \mathcal{L}}{\partial \phi_{i} \partial A_{\beta}}-\partial_{\nu}\left(\frac{\partial^{2} \mathcal{L}}{\partial\left(\partial_{\nu} \phi_{i}\right) \partial A_{\beta}}\right)-4 \varepsilon_{i k} \partial_{\beta} \phi_{k} \\
& 0=\quad+\left(\frac{\partial^{2} \mathcal{L}}{\partial \phi_{i} \partial\left(\partial_{\beta} \phi_{j}\right)}-\frac{\partial^{2} \mathcal{L}}{\partial\left(\partial_{\beta} \phi_{i}\right) \partial \phi_{j}}\right) \varepsilon_{j k} \phi_{k} \\
& -2 \varepsilon_{i k} \partial^{2} \phi_{k} \\
& 0=+\left(\frac{\partial^{2} \mathcal{L}}{\partial \phi_{i} \partial\left(\partial_{\beta} \phi_{j}\right)}-\frac{\partial^{2} \mathcal{L}}{\partial\left(\partial_{\beta} \phi_{i}\right) \partial \phi_{j}}\right) \varepsilon_{j k} \partial_{\beta} \phi_{k} \\
& +\left(\frac{\partial^{2} \mathcal{L}}{\partial \phi_{i} \partial \phi_{j}}-\partial_{\nu}\left(\frac{\partial^{2} \mathcal{L}}{\partial\left(\partial_{\nu} \phi_{i}\right) \partial \phi_{j}}\right)\right) \varepsilon_{j k} \phi_{k}
\end{aligned}
$$

For a constant solution to the $\phi$ equations of motion,i.e., $\phi=$ constant but $A_{\mu}$ unspecified, these equations reduce to

$$
\begin{gathered}
0=\frac{\partial^{2} \mathcal{L}}{\partial A_{\alpha} \partial A_{\beta}}+\frac{\partial^{2} \mathcal{L}}{\partial A_{\alpha} \partial\left(\partial_{\beta} \phi_{j}\right)} \varepsilon_{j k} \phi_{k} \\
0=\frac{\partial^{2} \mathcal{L}}{\partial A_{\alpha} \partial \phi_{j}} \varepsilon_{j k} \phi_{k} \\
0=\frac{\partial^{2} \mathcal{L}}{\partial\left(\partial_{\nu} \phi_{i}\right) \partial A_{\beta}}+2 g^{\nu \beta} \varepsilon_{i k} \phi_{k} \\
0=\left(\frac{\partial^{2} \mathcal{L}}{\partial \phi_{i} \partial A_{\beta}}-\partial_{\nu}\left(\frac{\partial^{2} \mathcal{L}}{\partial\left(\partial_{\nu} \phi_{i}\right) \partial A_{\beta}}\right)\right. \\
+\left(\frac{\partial^{2} \mathcal{L}}{\partial \phi_{i} \partial\left(\partial_{\beta} \phi_{j}\right)}-\frac{\partial^{2} \mathcal{L}}{\partial\left(\partial_{\beta} \phi_{i}\right) \partial \phi_{j}}\right) \varepsilon_{j k} \phi_{k} \\
0=\left(\frac{\partial^{2} \mathcal{L}}{\partial \phi_{i} \partial \phi_{j}}-\partial_{\nu}\left(\frac{\left.\partial_{\nu} \phi_{i}\right) \partial \phi_{j}}{2}\right)\right) \varepsilon_{j k} \phi_{k}
\end{gathered}
$$

Substituting (33) into (31) yields

$$
\frac{\partial^{2} \mathcal{L}}{\partial A_{\alpha} \partial A_{\beta}}=2 g^{\alpha \beta} \phi_{i} \phi_{i}
$$

and so for constant solutions to the equation, $\phi_{i}$, which are not zero, the gauge bosons must have a nonzero mass equal to $\sqrt{2 \phi_{i} \phi_{i}}$. The sign of the gauge boson mass is correct since the Lagrangian for massive vector bosons, the Proca Lagrangian, has the form $-\frac{1}{4} F^{2}+\frac{1}{2} M^{2} A^{2}$. Hence the first part of the Higgs mechanism, the gauge bosons acquire a mass, is automatic and can be seen to be simply a direct requirement of the generalized Goldstone's theorem, equation (6) or equivalently (7).

Applying $\varepsilon_{i l} \phi_{\mathrm{l}}$ to (34) equation and using (32) yields

$$
\partial_{\nu}\left(\frac{\partial^{2} \mathcal{L}}{\partial\left(\partial_{\nu} \phi_{i}\right) \partial A_{\beta}}\right) \varepsilon_{i l} \phi_{\mathfrak{l}}=0
$$


And applying $\varepsilon_{i \mathfrak{l}} \phi_{\mathfrak{l}}$ to (35) yields

$$
\varepsilon_{i l} \phi_{l} \frac{\partial^{2} \mathcal{L}}{\partial \phi_{i} \partial \phi_{j}} \varepsilon_{j k} \phi_{k}=\varepsilon_{i l} \phi_{l} \partial_{\nu}\left(\frac{\partial^{2} \mathcal{L}}{\partial\left(\partial_{\nu} \phi_{i}\right) \partial \phi_{j}}\right) \varepsilon_{j k} \phi_{k}
$$

These two equations along with (32) are automatically satisfied by taking

$$
\mathcal{L}_{A \phi}=2 A_{\mu} \varepsilon_{i j} \phi_{i} \partial^{\mu} \phi_{j}+\phi_{i} \phi_{i} A_{\mu} A^{\mu}
$$

which is the standard form.

The second part of the Higgs mechanism, the disappearance of the "wouldbe" Goldstone boson, follows from the symmetry condition 21 itself which after changing variables to $\xi$ and $\rho$ using $\phi=\rho e^{i \xi}$ reads

$$
0=\frac{\partial \mathcal{L}}{\partial\left(\partial_{\mu} A_{\nu}\right)} \partial_{\mu} \partial_{\nu} \theta+\left(\frac{\partial \mathcal{L}}{\partial A_{\nu}}+\frac{\partial \mathcal{L}}{\partial\left(\partial_{\nu} \xi\right)}\right) \partial_{\nu} \theta+\left(\frac{\partial \mathcal{L}}{\partial \xi}\right) \theta
$$

Again, since $\theta$ is an arbitrary function, each term must vanish separately. The first term vanishes due to the gauge invariance $\mathcal{L}_{A}$ since $\partial \mathcal{L} / \partial\left(\partial_{\mu} A_{\nu}\right)=$ $\partial \mathcal{L}_{A} / \partial\left(\partial_{\mu} A_{\nu}\right)$. The last term demands $\partial \mathcal{L} / \partial \xi=0$ and so $\mathcal{L}$ may depend only on derivatives of $\xi$, i.e., $\mathcal{L}(A, \rho, \partial \rho, \xi, \partial \xi) \rightarrow \mathcal{L}(A, \rho, \partial \rho, \partial \xi)$. If we make the change of variables $A_{\mu} \rightarrow B_{\mu}=A_{\mu}-\partial_{\mu} \xi$, the first and third terms still vanish. the first automatically since for $\mathcal{L}_{A}$ alone this is just a gauge tranformation and

the last still yields the condition $\partial \mathcal{L} / \partial \xi=0$. But now the middle term can be written as

$$
\begin{aligned}
0= & \left(\frac{\partial \mathcal{L}(B, \rho, \partial \rho, \partial \xi)}{\partial B_{\nu}}\right) \frac{\partial B_{\nu}}{\partial A_{\mu}}+\left(\frac{\partial \mathcal{L}(B, \rho, \partial \rho, \partial \xi)}{\partial B_{\nu}}\right) \frac{\partial B_{\nu}}{\partial\left(\partial_{\mu} \xi\right)} \\
& +\left(\frac{\partial \mathcal{L}(B, \rho, \partial \rho, \partial \xi)}{\partial\left(\partial_{\nu} \xi\right)}\right) \\
= & \left(\frac{\partial \mathcal{L}(B, \rho, \partial \rho, \partial \xi)}{\partial B_{\nu}}\right) \delta_{\nu}^{\mu}+\left(\frac{\partial \mathcal{L}(B, \rho, \partial \rho, \partial \xi)}{\partial B_{\nu}}\right)\left(-\delta_{\nu}^{\mu}\right) \\
& +\left(\frac{\partial \mathcal{L}(B, \rho, \partial \rho, \partial \xi)}{\partial\left(\partial_{\nu} \xi\right)}\right) \\
= & \frac{\partial \mathcal{L}(B, \rho, \partial \rho, \partial \xi)}{\partial\left(\partial_{\nu} \xi\right)}
\end{aligned}
$$

and hence $\mathcal{L}$ does not depend on $\partial \xi$ and so the "would-be" Goldstone boson $\xi$ has completely vanished from the model. Effectively it has become the longitudinal component of a now massive gauge boson.

\section{$5 \quad$ Scale and Conformal Symmetry Breaking}

It has been noted in the literature, [国, see also[5], that in theories with spontaneously broken scale and conformal invariance, although five symmetries are broken, only one Goldstone boson appears. A similar thing occurs for broken 
Lorentz invariance in a class of three-dimensional gauge theories as discussed in [?]. In this section we use our more general treatment of Goldstone's theorem to study this question. In particular, we see that our equation predicts only one Goldstone mode, but also imposes four other conditions, not having to do with particle masses, that represent the extra information contained in the spontaneous breakdown of conformal symmetry.

We have in mind a model of the kind considered by Coleman, which contains a scalar and a fermion field, and another scalar, the dilaton, whose role is to implement the broken symmetry. Since we do not consider fermions in this paper, we shall omit them here. Also, we note that the extension of the following discussion to include more then one scalar (but still only one dilaton) is straightforward, but to keep our notation simple we do not put them in explicitly.

Here $\phi_{i}$ will be a doublet: $\phi_{i}=\left[\begin{array}{c}\phi \\ \sigma\end{array}\right]$, where $\phi$ is the ordinary scalar field and $\sigma$ is the dilaton. Under dilations, they transform as

$$
\delta \phi=\phi+x^{\mu} \partial_{\mu} \phi
$$

and

$$
\delta \sigma=\frac{1}{f}+x^{\mu} \partial_{\mu} \sigma
$$

where $f$ is a scale characterizing the symmetry breaking. Under special conformal transformations, we have:

$$
\begin{aligned}
& \delta^{\lambda} \phi=\left(2 x^{\lambda} x^{\rho}-g^{\lambda \rho} x^{2}\right) \partial_{\rho} \phi+2 x^{\lambda} \phi \\
& \delta^{\lambda} \sigma=\left(2 x^{\lambda} x^{\rho}-g^{\lambda \rho} x^{2}\right) \partial_{\rho} \sigma+\frac{2 x^{\lambda}}{\mathrm{f}} .
\end{aligned}
$$

In what follows, we shall assume that translation invariance is not broken. Hence $\phi$ and $\sigma$ must be constants. However, one sees that the $\Delta$ 's will not be constants. We have, in fact,

$$
\delta \phi=\phi, \quad \delta \sigma=\frac{1}{\mathrm{f}} \quad \text { (dilations) }
$$

but

$$
\left.\delta^{\lambda} \phi=2 x^{\lambda} \phi, \quad \delta^{\lambda} \sigma=\frac{2 x^{\lambda}}{\mathrm{f}} \quad \text { (conformal transfs. }\right) .
$$

Noting further that the spacetime derivatives of the Lagrangian density $\mathcal{L}$ or of its derivatives with respect to the fields will vanish, we obtain from (7),

$$
\left[\begin{array}{ll}
\frac{\partial^{2} \mathcal{L}}{\partial \phi^{2}} & \frac{\partial^{2} \mathcal{L}}{\partial \phi \partial \sigma} \\
\frac{\partial^{2} \mathcal{L}}{\partial \sigma \partial \phi} & \frac{\partial^{2} \mathcal{L}}{\partial \sigma^{2}}
\end{array}\right]\left[\begin{array}{l}
\phi \\
1 / \mathrm{f}
\end{array}\right]=0 \quad \text { (dilations) }
$$

and

$$
\left(x^{\lambda}\left[\begin{array}{cc}
\frac{\partial^{2} \mathcal{L}}{\partial \phi^{2}} & \frac{\partial^{2} \mathcal{L}}{\partial \phi \sigma} \\
\frac{\partial^{2} \mathcal{L}}{\partial \sigma \partial \phi} & \frac{\partial^{2} \mathcal{L}}{\partial \sigma^{2}}
\end{array}\right]+\left[\begin{array}{cc}
0 & \frac{\partial^{2} \mathcal{L}}{\partial \phi \partial\left(\partial_{\lambda} \sigma\right)}-\frac{\partial^{2} \mathcal{L}}{\partial \sigma \partial\left(\partial_{\lambda} \phi\right)} \\
\frac{\partial^{2} \mathcal{L}}{\partial \sigma \partial\left(\partial_{\lambda} \phi\right)}-\frac{\partial^{2} \mathcal{L}}{\partial \phi \partial\left(\partial_{\lambda} \sigma\right)} & 0
\end{array}\right]\right)\left[\begin{array}{l}
\phi \\
1 / \mathrm{f}
\end{array}\right]=0
$$


(conformal transfs.)

In the second equation, the two terms must separately vanish, because the first is proportional to the variable $x^{\lambda}$ and the second is not. But the first term encodes exactly the same information as does equation (46). This is the origin of the fact that dilations and special conformal transformations give rise to the same Goldstone boson. There is, however, the second term in (47), which provides an additional set of four constraints:

$$
\frac{\partial^{2} \mathcal{L}}{\partial \sigma \partial\left(\partial_{\lambda} \phi\right)}=\frac{\partial^{2} \mathcal{L}}{\partial \phi \partial\left(\partial_{\lambda} \sigma\right)}
$$

This is, in principle, the "extra" information about the Lagrangian (or the effective action, when quantum corrections are considered) that follows from spontaneously broken conformal symmetry.

Let us see how this works at tree level in the specific model considered by Coleman. The Lagrange density is

$$
\mathcal{L}=\frac{1}{2} \partial_{\mu} \phi \partial^{\mu} \phi+\frac{1}{2 f^{2}} \partial_{\mu}\left(e^{f \sigma}\right) \partial^{\mu}\left(e^{f \sigma}\right)-\frac{\mu^{2}}{2} \phi^{2} e^{2 f \sigma}-\frac{\lambda}{4 !} \phi^{4} .
$$

The equations (46) and (47) imply

$$
\left[\begin{array}{ll}
1+\frac{\lambda}{2 \mu^{2}} \phi^{2} e^{-2 f \sigma} & 2 \mathrm{f} \phi \\
2 \mathrm{f} \phi & 2 \mathrm{f}^{2} \phi^{2}
\end{array}\right]\left[\begin{array}{l}
\phi \\
1 / \mathrm{f}
\end{array}\right]=0
$$

which requires $\phi=0$ and identifies $\left[\begin{array}{l}0 \\ 1 / \mathrm{f}\end{array}\right]$ (i.e. the $\sigma$ particle) as the Goldstone mode. The extra information furnished in eq. (47) is trivial in this case, since the relevant terms were set to zero from the beginning.

Acknowledgements. The work of A.C. was supported in part by DOE grant \#FG02-92ER-40704.

\section{References}

[1] Michael E. Peskin and Daniel V. Schroeder, "An Introduction to Quantum Field Theory", Perseus Publishing, 1994.

[2] Steven Weinberg, "The Quantum Theory of Fields", Volume 2, Cambridge University Press, 1996.

[3] C Itzykson and J-B. Zuber, "Quantum Field Theory", McGraw-Hill, 1980.

[4] S. Coleman, "Aspects of Symmetry", Cambridge University Press, 1990.

[5] J. Polchinski, Nuclear Physics B303, 226 (1988); J. Polchinski, 1992 TASI Lectures (hep-th/9210046), footnote 6 .

6] Y. Hosotani, Proceedings DPF94, p. 1403 (QCD161,A6,1994)(hepth/9407188) 